\title{
Gangrena digital em extremidade superior secundária à infusão intravenosa local de vancomicina: relato de caso
}

\author{
Clóvis Luís Konopka*, Mariana Rechia Bitencourt**, Luciane \\ Durigon Cocco***, Diogo Chagas Dalcin**, Anderson Kahl Beck**, \\ Tiago Dani**, Marçal Roumow**
}

Resumo: Em decorrência de sua crescente recomendação para tratamento de infecções hospitalares por Staphylococcus sp. com resistência à meticilina e oxacilina, a utilização do antibiótico glicopeptídeo vancomicina aumentou muito nos últimos 20 anos. Apesar desde fato, o número de pacientes que eventualmente apresenta efeitos adversos graves à sua administração é muito pequeno, sendo mais frequentes reações simples como dor, tromboflebite no local de administração, febre e calafrios. Entre as complicações graves relacionadas ao seu uso está a anafilaxia, a necrólise epidérmica tóxica, 0 eritema multiforme, a ototoxicidade e a 'síndrome do homem vermelho'. Neste artigo descrevemos um caso muito raro e excepcional de gangrena isquêmica de todos os quirodáctilos da mão direita após infusão intravenosa local de vancomicina.

Descritores: Vancomicina, Efeitos adversos, Toxicidade, Gangrena.

\section{Digital gangrene in the upper extremity secondary to local intravenous infusion of vancomycin: case report}

\begin{abstract}
The use of the glycopeptide antibiotic vancomycin has increased in the last 20 years, due to its growing recommendation for the treatment of nosocomial infections caused by staphylococci $\mathrm{sp}$. resistant to methicillin and oxacillin. The number of patients who may eventually present serious adverse side effects is very small, with simple reactions more frequent as pain and thrombophlebitis at the injection site, fever and chills. Among the serious complications are reported anaphylaxis, toxic epidermal necrolysis, erythema multiforme, ototoxicity and 'red man syndrome'. In this article we describe a very rare and exceptional case of ischemic gangrene on all fingers of the right hand after local intravenous infusion of vancomycin.
\end{abstract}

Descriptors: Vancomycin, Adverse effects, Toxicity, Gangrene.

\footnotetext{
*Médico pela Universidade Federal de Santa Maria (UFSM), Santa Maria, RS, Brasil. Docente de Cirurgia Vascular no Hospital Universitário de Santa Maria (HUSM), Santa Maria, RS, Brasil.

**Acadêmico de Medicina pela Universidade Federal de Santa Maria (UFSM), Santa Maria, RS, Brasil.

${ }^{* \star *}$ Médica pela Universidade Federal de Santa Maria (UFSM), Santa Maria, RS, Brasil. Residente de Cardiologia em Instituto de Cardiologia de Porto Alegre, Porto Alegre, RS, Brasil.
} 


\section{Introdução}

A vancomicina é um antibiótico glicopeptídeo originalmente isolado pelo laboratório "Eli Lilly and Company" a partir da fermentação por bactérias de um membro da família Actinomicetes, Amycolatopsis orientalis. ${ }^{1-3} 0$ composto tem seu mecanismo de ação baseado primariamente na inibição da biossíntese da parede celular bacteriana. Este achado resultou da observação nas pesquisas iniciais do efeito desta substância sobre a bactéria Staphylococcus aureus, que não desenvolvia resistência significativa após contatos seriados com meios de cultura contendo esta droga. Deste modo, a vancomicina teve como indicação original o tratamento de infecções por Staphylococcus aureus, tendo sido aprovada pelo Food and Drug Administration (FDA) dos Estados Unidos em 1958 para essa finalidade, e lançada no mercado brasileiro posteriormente com o nome comercial de Vancocina®. ${ }^{3}$

Atualmente, o uso da vancomicina é reservado para o tratamento de infecções estafilocócicas sistêmicas em pacientes alérgicos aos $\beta$-lactâmicos, infecções por estafilococos resistentes à oxacilina, enterococos e pneumococos resistentes à penicilina, além de representar uma alternativa para o tratamento da enterocolite por Clostridium difficile. ${ }^{4} \mathrm{O}$ grande número de publicações recentes sobre a vancomicina reflete 0 aumento significativo do seu uso nos últimos 30 anos e o interesse do meio científico em compreender melhor as suas indicações e principalmente o uso correto desse importante antibiótico no arsenal terapêutico antimicrobiano nos dias de hoje.

As reações adversas significativas ou graves já relatadas em associação ao uso desta droga como: anafilaxia, necrólise epidérmica tóxica, eritema multiforme, ototoxicidade, nefrotoxicidade e a 'síndrome do homem vermelho' são proporcionalmente incomuns. No entanto, reações agudas menos intensas são corriqueiras e relativamente freqüentes incluindo dor e tromboflebite no local de aplicação, rash máculo-papular, febre, calafrios e prurido. ${ }^{4-5}$

Neste artigo descrevemos um caso excepcional, jamais descrito na literatura revisada através de buscas no MedLine/SCieLo (palavras-chave: Vancomycin digital gangrenous, drug induced digital grangrenous, Uncommon Vancomycin-Induced Side Effects); de uma complicação grave e mutiladora como a gangrena isquêmica de todos os quirodáctilos da mão direita com necessidade de amputação, após infusão intravenosa (IV) local de vancomicina.

\section{Metodologia}

Este artigo trata de um relato de caso, realizado através de análise retrospectiva de prontuário médico. Revisão bibliográfica foi feita a partir de pesquisa no MedLine/SCieLo com as palavras-chave: Vancomycin digital gangrenous, drug induced digital grangrenous, Uncommon Vancomycin-Induced Side Effects.

\section{Relato de caso}

Saúde (Santa Maria), v.38, n.2, p. 77-84, 2012. Gangrena digital em extremidade superior secundária à infusão intravenosa local de vancomicina: relato de

Paciente homem, 71 anos, branco, agricultor, ex-tabagista de 30 maços-ano, com diagnóstico de adenocarcinoma pulmonar (estádio IA), submetido à lobectomia pulmonar inferior esquerda acompanhada de ressecção de linfonodos hilares e mediastinais. No sexto 
dia de pós-operatório iniciou com quadro de dispnéia, confusão mental e febre com leucocitose, sendo diagnosticado à radiografia de tórax um derrame pleural à esquerda. Foi submetido à toracocentese com aspiração de líquido espesso de aspecto purulento, estabelecendo o diagnóstico de empiema. Tratado, inicialmente, com drenagem torácica intercostal fechada em selo d'água. A cultura inicial da secreção demonstrou a presença de $S$. aureus meticilina-resistente (MRSA), e, então, foi iniciada a administração de vancomicina na dose de $15 \mathrm{mg} / \mathrm{kg}$ a cada 12 horas IV, através de venóclise no dorso da mão direita.

No $2^{\circ}$ dia de utilização dessa droga e durante a fase inicial de infusão (< 30 minutos), 0 paciente referiu dor intensa em toda a mão direita, acompanhada de parestesias associadas à palidez e à friabilidade, com pulso radial e cubital presentes. Não foram observados sinais sistêmicos do tipo alérgico ou de extravasamento local da droga, bem como não havia relatos anteriores de tromboflebite ou de qualquer anormalidade com a referida extremidade. A via de administração teve sua patência testada, funcionando adequadamente, não sendo observado trajeto no subcutâneo. Também não foram realizadas infusões de outras drogas no mesmo acesso venoso, apenas injeção de soro fisiológico 0,9\%.

A administração de Vancomicina foi imediatamente descontinuada e devido ao vasoespasmo persistente, foi iniciada a administração IV sistêmica de alprostadil associada à anticoagulação plena com heparina não fracionada, com ajuste pelo tempo de tromboplastina parcial ativada (TTPA). Após 24 horas foi observada reversão quase completa da palidez e da frialdade, exceto nos quirodáctilos, os quais evoluíram nos dias subsequentes para cianose fixa e posteriormente para gangrena seca como observado nas figuras 1 e 2 (Fig. 1 e Fig. 2). Cerca de duas semanas após o início do quadro o paciente foi submetido à amputação de todos os quirodáctilos desta mão conforme visto na figura 3 (Fig.3). No $8^{\circ}$ dia após esta cirurgia, o paciente evoluiu ao óbito em decorrência de extenso infarto agudo do miocárdio.

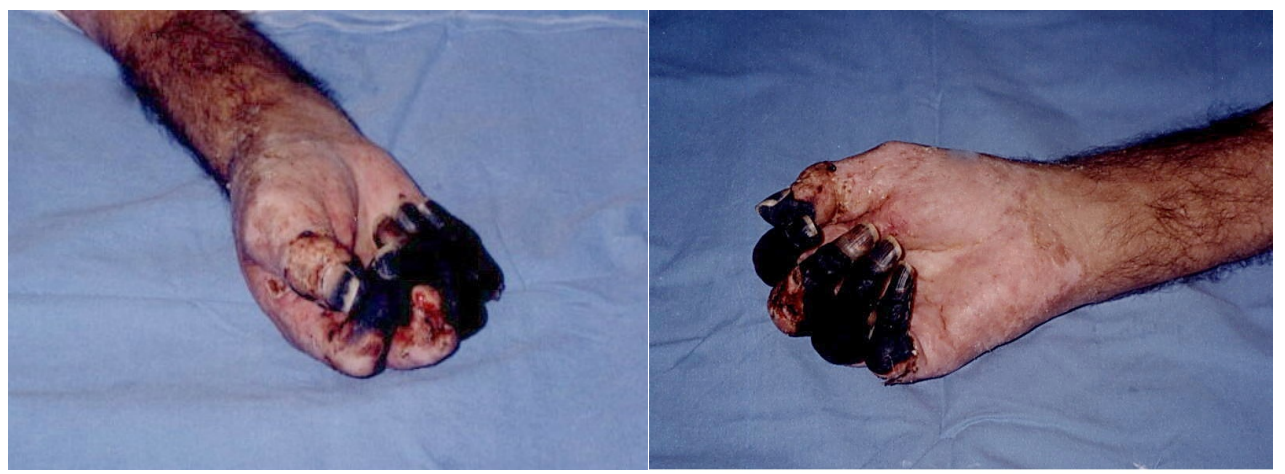

Fig. 1 e Fig. 2 - Gangrena isquêmica com mumificação de todos os quirodáctilos da mão direita.

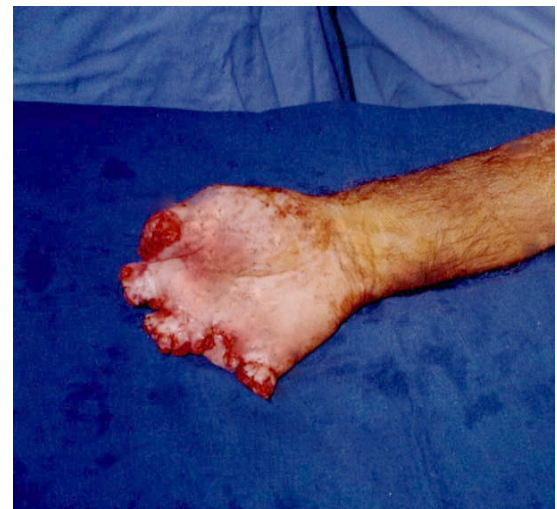

Saúde (Santa Maria), v.38, n.2, p. 77-84, 2012. Konopka, C. L., et al.

Fig. 3 - Pós-operatório imediato de amputação de quirodáctilos da mão direita. 


\section{Discussão}

Eventos adversos a medicamentos em pacientes hospitalizados representam uma patologia emergente associada a um significante aumento nos dias de internação, nos custos e na morbidade. Bates et al estimaram em aproximadamente dois mil dólares o custo adicional associado aos pacientes hospitalizados com evento adverso, e o aumento de aproximadamente 1,9 dias na permanência hospitalar. ${ }^{6}$

Os antibióticos estão entre os medicamentos mais prescritos em hospitais, responsáveis por $20 \%$ a $50 \%$ dos gastos com medicamentos, além de serem apontados como um dos grupos medicamentosos que mais causam efeitos adversos. ${ }^{7}$

O antibiótico glicopeptídeo vancomicina apresenta vários mecanismos de ação, sendo 0 mais importante a inibição da síntese da parede celular e sua destruição por lise. Esta droga é especialmente útil no tratamento de infecções graves causadas por microrganismos suscetíveis, em especial cocos e bacilos gram-positivos, como estafilococos, enterococos e pneumococos. ${ }^{2-4}$ A partir da década de 1980, a vancomicina passou a apresentar uma enorme relevância clínica devido ao surgimento, cada vez mais comum, de pacientes com infecções hospitalares graves por cepas de estafilococos meticilina/oxacilina-resistentes (MRSA) e enterococos ampicilina-resistentes. Entretanto, existem estudos limitados sobre os padrões farmacocinéticos desta droga neste grupo particular de pacientes. Com este súbito aumento do uso, foi grande a efervescência de relatos de reações adversas comuns e outras jamais descritas em decorrência de sua infusão.

A vancomicina não é absorvida adequadamente por via oral (VO) e a sua administração intramuscular (IM) pode provocar dor, hipersensibilidade e necrose local por ser extremamente irritante aos tecidos devido principalmente ao seu pH variar dentre 2,8 a 4,5. ${ }^{4}$ Por esse motivo, recomenda-se realizar sua administração sob diluição por via IV, na infusão de até $10 \mathrm{mg} / \mathrm{min}$, em período de tempo nunca inferior a 60 minutos. ${ }^{4}$ Devido à complexa farmacocinética, o monitoramento de sua concentração sérica deve ser realizado, visando manter níveis dentre 5 a $10 \mathrm{mg} / \mathrm{L}$.4, 8 E embora seja considerada segura em níveis terapêuticos, o emprego de concentrações maiores ou em menor tempo podem aumentar 0 risco de reações relacionadas com a sua infusão.

Estima-se que 5 a $15 \%$ dos pacientes tratados com algum medicamento desenvolvam reações adversas, sendo que 2 a $3 \%$ das reações constituem-se em reações cutâneas. ${ }^{9} \mathrm{~A}$ ocorrência de reações adversas aos antibióticos é, também, muito comum, porém, o número de pacientes que apresenta efeitos adversos significativos ou graves com 0 uso da vancomicina é relativamente muito pequeno. Em contraste a este dado, reações agudas menos graves são relativamente frequentes, observadas em torno de $10 \%$ do total de aplicações. Tais reações incluem febre e rash (1-3\%), dor local, tromboflebite (13\%), rash máculo-papular, febre, calafrios e prurido. ${ }^{4} \mathrm{Em}$ cerca de 1-10\% dos casos se observa a presença da denominada síndrome do homem vermelho, considerada a mais comum toxicidade da terapia com a vancomicina. ${ }^{4} \dot{E}$ caracterizada clinicamente por um eritema ou rash maculopapular em face, tórax e extremidades superiores, algumas vezes acompanhados de hipotensão, prurido e angioedema de face, sendo mais comum devido à

Saúde (Santa Maria), v.38, n.2, p. 77-84, 2012. Gangrena digital em extremidade superior secundária infusão rápida da droga ( $>500 \mathrm{mg}$ em tempo inferior a 30 minutos). Frequentemente, este quadro é confundido como uma reação alérgica ou anafilactóide relacionada a uma descarga aguda de histamina, porém os pacientes usualmente toleram as próximas doses se a diluição e o período de infusão forem aumentados. ${ }^{5}$ 
Relatos isolados de neutropenia $(2 \%)$ e eosinofilia associada à vancomicina são encontrados na literatura, bem como casos de agranulocitose e trombocitopenia, provavelmente relacionados com mecanismos de destruição imunológica. ${ }^{10} \mathrm{Em}$ alguns casos, a vancomicina pode produzir nefrite intersticial, ototoxicidade, dermatose bolhosa IgA linear, eritroderma esfoliativo, síndrome de DRESS (drug rash eosinophilia with systemic symptoms), vasculite cutânea necrotizante e necrólise epidérmica tóxica. ${ }^{1,5,11,12,13}$ Também já foram descritos casos de Síndrome de Stevens-Johnson. ${ }^{11,14}$

Uma situação incomum e peculiar à administração IM de certas drogas é conhecida como Embolia Cutis Medicamentosa ou Síndrome de Nicolau. Confluente em partes ao presente caso relatado, caracteriza-se por dor intensa no local da administração, seguida por descoloração da pele no mesmo sítio, podendo evoluir para necrose de pele e tecidos profundos. ${ }^{15}$ Apesar da descrição de casos desta síndrome após injeção IM de vitamina K, antiinflamatórios não-esteróides, vacinas, metoclopramida, noradrenalina, quimioterápicos, penicilina benzatina e penicilina $G$, casos conseqüentes à administração por via EV de vancomicina jamais foram descritos na literatura médica. ${ }^{15-17}$

A etiopatogenia desta síndrome não é totalmente esclarecida, existindo hipóteses de vasoespasmo secundário a picada da agulha, embolização do material injetado ou compressão vascular mecânica pela droga injetada. A origem multifatorial da lesão local permitiu a Barthélemy postular a existência de quatro diferentes tipos de dermatite livedoide: 1) o exantema embólico local; 2) a placa equimótica e flictenular; 3) a dermite livedoide e granulosa; 4) a gangrena profunda hipodérmica e muscular. Ele não fez referências às lesões de mão do presente relato. ${ }^{18} \mathrm{~A}$ recomendação de tratamento para esta condição é baseado na rápida instituição de medidas para a contenção da progressão das lesões, e incluem 0 oxigênio hiperbárico, alprostadil intravenoso e anticoagulação com heparina, porém não há diretrizes estabelecidas para o manejo destes casos. ${ }^{15-16}$

Em nosso caso, o paciente não apresentou qualquer tipo de reação imediata à infusão da primeira dose da droga, nem sinais de hiperssensibilidade sistêmicos ou locais. Também não apresentava sinais clínicos prévios de doença arterial, sendo o único indicativo a sua carga tabágica(30 maços-ano). Na literatura, são encontrados relatos de gangrena induzida por quimioterápicos e Talidomida admnistrados IV, e por outras drogas administradas IV acidentalmente. ${ }^{24}$ Bem como a síndrome de Nicolau, que é ocasionada por administração de certas medicações por via IM, esse é o primeiro relato de isquemia arterial aguda induzida pela administração intravenosa de vancomicina. Necessitamos observar atentamente à ocorrência de novos casos e buscar uma melhor elucidação do seu real mecanismo.

Os efeitos colaterais da vancomicina, em geral, devem ser tratados com a suspensão imediata de sua infusão, reservando o uso de anti-histamínicos e corticosteróides para os casos mais graves, de natureza alérgica ou anafilática. ${ }^{17}$

\section{Considerações finais}

Esse é o relato de uma complicação inédita de isquemia arterial aguda induzida pela administração intravenosa de vancomicina. Perante esse quadro faz-se mister alertar para sua ocorrência, bem como para a vigilância perante novos casos. Sabemos que todas as

Saúde (Santa Maria), v.38, n.2, p. 77-84, 2012. Konopka, C. L., et al. ISSN $2236-5843$ drogas têm um potencial para reações adversas, portanto deve-se considerar o risco- 
benefício antes de sua administração. Pacientes submetidos à terapia com vancomicina devem receber especial atenção durante e após o processo de infusão da droga, a fim de se detectar precocemente quaisquer alterações e reduzir ao máximo seus efeitos adversos e suas consequências.

\section{Referências}

1. Shnayerson M, Plotkin M. The Killers Within: The Deadly Rise of Drug-Resistant Bacteria. $1^{\text {th }}$ ed. Back Bay Books; 2003. 44-57p

2. Levine D. Vancomycin: a history. Clin Infect Dis. 2006; 42 (1): S5-S12.

3. De Almeida R. Vancomicina: Avaliação do uso em pacientes internados na unidade de terapia intensiva. Porto Alegre: 2011. 13p. Dissertação (Mestrado) - Universidade Federal do Rio Grande Do Sul, 2011.

4. Agência Nacional de vigilância Sanitária. Bulário eletrônico. Brasil: 2009 [citado em: 25 nov 2010].Disponivel em: http://www4.anvisa.gov.br/base/visadoc/BM/BM[26312-1-0].PDF

5. Vidal C, Quintela AG, Fuente R. Toxic epidermal necrolysis due to vancomycin. Ann Allergy 1992; 68: 3457

6. Bates DW, Spell N, Cullen DJ, Burdick E, Laird N, Petersen LA, et al. The costs of adverse drug events in hospitalized patients. Adverse Drug Events Prevention Study Group. JAMA. 1997; 277(4): 307-11.

7. Vlahovic-Palcevski V, Morovic M, Palcevski G. Antibiotic utilization at the university hospital after introducing an antibiotic policy. Eur J Clin Pharmacol. 2000; 56(1): 97-101.

8. Logsdon BA, Lee KR, Barret FF. Correct dosing of vancomycin in infants and children. Pediatrics. 1995; 96:1177.

9. Tozato C, De Alcantara M, Pasternak J. Reações cutâneas provavelmente atribuídas ao uso de fenitoína e vancomicina. Einstein. 2008; 6(2): 200-2

10. Zenon GJ, Cadle RM, Hamill RJ. Vancomycin-induced thrombocytopenia. Arch Intern Med 1991; 151: 995-6.

11. Laurencin CT, Horan RF, Senatus PB, Wheeler CB, Lipson SJ. Stevens-Johnson- type reaction with vancomycin treatment. Ann Pharmacother. 1992; 26: 1250-1

12. Tamagawa-Mineoka R, Katoh N, Nara T, Nishimura $\mathrm{Y}$, Yamamoto S,Kishimoto S. DRESS syndrome caused by teicoplanin and vancomycin, associated with reactivation of human herpesvirus-6. Int J Dermatol. 2007; 46: 654-5.

13. Kwon HS, Chang YS, Jeong YY, Lee SM, Song WJ, Kim HB et al. A case of hypersensitivity syndrome to both vancomycin and teicoplanin. J Korean Med Sci. 2006; 21:1108.

14. Alexander II, Greenberger PA. Vancomycin-induced Stevens-Johnson syndrome. Allergy Asthma Proc. $1996 ; 17: 75-8$.

15. Nischal KC, Basavaraj HB, Swaroop MR, Agrawal DP, Sathyanarayana BD, Umashankar NP. Nicolau Syndrome: An latrogenic Cutaneous Neclosis. J Cutan Aesthet Surg .2009; 2: 92-5

16. Roszell S, Jones C. Intravenous administration issues: a comparison of intravenous insertions and complications in vancomycin versus other antibiotics. J Infus Nurs. 2010; 33(2):112-8.

Saúde (Santa Maria), v.38, n.2, p. 77-84, 2012. Gangrena digital em extremidade superior secundária à infusão intravenosa local de vancomicina: relato de

17. Duque FL V, Chagas CAA. Acidente por injeção medicamentosa no músculo deltóide: lesões locais e à distância, revisão de 32 casos. J Vasc Bras. 2009; 8(3): 238-46. 
18. Barthélemy R. Embolie artérielle par le bismuth liposolubile. Bull Soc Fr Derm Syph. 1930; 37:1025-7.

19. Marinho DS, Huf G, Ferreira BLA, Castro H, Rodrigues CR, Sousa VP et al. The study of vancomycin use and its adverse reactions associated to patients of a Brazilian university hospital. BMC Research Notes. 2011; 4: 236

20. An S, Hwang E, Kim J, Jin H, Jin S, Kyun J et al, Vancomycin-Associated Spontaneous Cutaneous Adverse Drug Reactions. Allergy Asthma Immunol Res. 2011;3(3):194-198.

21. Craycraft ME, Arunakul VL, Humeniuk JM. Probable vancomycin-associated toxic epidermal necroslysis. Pharmacotherapy. 2005; 25: 308-12.

22. Center for Disease Control. Recommendations for Preventing the Spread of Vancomycin Resistance. Recommendations of the Hospital Infection Control Practices Advisory Committee (HICPAC). MMWR. 1995; 44(RR-12):1-13.

23. Moellering RC Jr. Vancomycin: a 50-year reassessment. Clin Infect Dis. 2006; 42 (1): S3-S4.

24. Kang TY, Lowe CJ, Sekeres MA. Thalidomide Use and Digital Gangrene. J Clin Oncol. 2006,42(33): 5328-5333

\section{Clóvis Luís Konopka}

Endereço para correspondência - Rua Duque de Caxias, 1668/803. Bairro Centro - Santa Maria - RS. CEP 97015-190

E-mail: cloviskonopka@gmail.com

Currículo Lattes: http://lattes.cnpq.br/0367178199063341

Recebido em 01 de setembro de 2012.

Aprovado em 30 de novembro de 2012. 
Saúde (Santa Maria), v.38, n.2, p. 77-84, 2012. Gangrena digital em extremidade superior secundária

à infusão intravenosa local de vancomicina: relato de

84

ISSN 2236-5834 